\title{
Atenolol - Scopolamine Combination Drug Decreases Acute Anxiety Post Trauma Therapy Session
}

Benjamin $\mathbf{A}^{\mathbf{1}^{*}}$, Mollner $\mathbf{A}^{2}$ and Dooley $\mathbf{T P}^{3}$

${ }^{1}$ Sterling Care Psychiatric Group in Ventura County, USA

${ }^{2}$ Seasons in Agoura, USA

${ }^{3}$ Trends in Pharma Development LLC, USA

*Corresponding author: Ashley Benjamin, Sterling Care Psychiatric Group in Ventura County, 4935 Via Camino, Newbury Park, CA 91320, USA

Received: September 01, 2017; Accepted: September 26, 2017; Published: October 03, 2017

\begin{abstract}
Anxiety symptoms often occur following psychotherapy sessions in patients with histories of trauma or Post-Traumatic Stress Disorder (PTSD). These patients can require an "as needed" (prn) medication to diminish their anxiety symptoms following therapy. The choice of FDA-approved prn anxiolytics is limited to benzodiazepines, buspirone, and hydroxyzine. However, benzodiazepines have the potential of addiction and abuse, and the other drugs are considered by many physicians to have limited efficacy. A PTSD patient was treated with medications and psychotherapy in a residential setting. However, she encountered severe symptoms of anxiety after psychotherapy sessions and required a prn treatment for acute anxiety following psychotherapy, for which a benzodiazepine was not suitable. A new patented anxiolytic was selected that is a combination of the beta blocker atenolol plus the antimuscarinic agent scopolamine to suppress the sympathetic and parasympathetic/CNS symptoms of acute anxiety, respectively. Therefore, she was treated with a PanX® drug combination of atenolol - scopolamine $\mathrm{HBr}$, and her acute anxiety symptoms were assessed pre- and post-treatment on three separate occasions. The patient's breakthrough anxiety was treated effectively with the new anxiolytic drug combination. She experienced a rapid calming effect within 25-30 minutes, that persisted for 3-4 hours, and affected both sympathetic and parasympathetic/CNS symptoms. The drug combination sufficiently decreased the PTSD patient's acute anxiety symptoms to enable the patient to continue psychotherapy for historic trauma. This is the first reported case of any beta blocker - antimuscarinic drug combination treatment of anxiety following psychotherapy.
\end{abstract}

Keywords: Psychotherapy; Beta blocker; Muscarinic; Adrenergic; Anxiolytic; PTSD

\section{Introduction}

It is a well-known phenomenon that anxiety often spikes post psychotherapy sessions. Often, the topics involving trauma, life transitions, grief or deep rooted issues result in short term postsession anxiety, which can sometimes derail the therapeutic progress. One study suggests that this rate of increased anxiety and stress is as high as 33 percent [1]. Often, this anxiety is short lived. However, for some patients, calming techniques are not adequate to allow the patient to function post therapy session. There is a dearth of research regarding this specific topic, but in general, the benzodiazepines have been the mainstay of treatment for acute anxiety.

The issue arises when patients have a benzodiazepine addiction, or abuse background, or are on opioids, what are other options that might alleviate the anxiety, without increasing a risk of benzodiazepine abuse or possible respiratory depression and death when combined with opioids? There is a strong correlation between mood/anxiety disorders and prescription opioid use [2]. Added to this dilemma is the unmet need in the current formulary for "as needed" (prn) anxiolytic drug treatments that are effective, fast-acting, and nonaddicting.

Here we introduce a new class of anxiolytic medicines that were specifically designed for the prn symptomatic relief of acute anxiety, yet without using any addictive benzodiazepines or other Controlled Substances. The recently patented PanX drug combinations contain a beta blocker plus an antimuscarinic agent [3]. The beta blocker inhibits epinephrine binding to address the sympathetic cardiovascular symptoms, most notably tachycardia and palpitations [3]. The antimuscarinic agent inhibits acetylcholine binding to address the parasympathetic and CNS symptoms, such as anxiousness, fear, avoidance, nausea, vomiting, and sweating [3].

Here we present a case report of the beneficial use of atenolol plus scopolamine in a PTSD patient with a polysubstance dependence background, who experienced breakthrough anxiety post psychotherapy.

\section{Case Presentation}

Patient $\mathrm{Z}$ is a 23 year old Caucasian female who was admitted to a dual diagnosis residential facility (Seasons in Malibu). She had previously been detoxed at another facility and was 8 days abstinent from a combination of alcohol and cocaine and four days abstinent from marijuana, heroin, and methamphetamines. She was evaluated by the Internal Medicine/Addiction Specialist, whose evaluation reflected she was fully detoxed. He maintained her on Prozac (fluoxetine) $40 \mathrm{mg}$ once in the AM for PTSD, depression, and anxiety symptoms, Seroquel (quetiapine) $25 \mathrm{mg}$ once at night to help with 
Table 1: Blood pressures and (Heart Rates) during three anxiety episodes and treatments with atenolol - scopolamine $\mathrm{HBr}$.

\begin{tabular}{|c|c|c|c|}
\hline Anxiety Episode & 1 & 2 & 3 \\
\hline Pre-treatment & $106 / 65(73)$ & $99 / 61(65)$ & $98 / 72(93)$ \\
\hline 1 hour Post-treatment & $94 / 62(61)$ & $98 / 57(47)$ & $99 / 61(67)$ \\
\hline 4 Hour Post-treatment & $86 / 58(60)$ & $98 / 65(68)$ & $98 / 69(86)$ \\
\hline
\end{tabular}

sleep, and prazosin $2 \mathrm{mg}$ once at night to eliminate nightmares. As the consulting psychiatrist, I (first author) saw her on the same day. Her story is as follows:

Patient $\mathrm{Z}$ has a family history of both addiction and depression. She denied any mental health symptoms until a sexual assault at age 16. Despite some treatment in a victims group, she endorsed a chronic history of recurrent memories, flashbacks, and nightmares. She also endorsed avoidance of these feelings and memories as well as worsening self-worth, self-blame, shame, guilt, feelings of detachment, and loss of positive emotions. She also experienced irritability, hyper vigilance, startle reflex, and difficulty getting and staying asleep. As part of her post-traumatic stress disorder, she experienced depression, generalized anxiety, and panic attacks that were situational to feelings or thoughts linked to the trauma. Mania, psychosis, Obsessive Compulsive Disorder, attention deficit, and eating disorders were ruled out. Her medical history was noncontributory. She denied any self-harm or suicidality history as she said, "I want to live and just be normal".

She did experience a chaotic early home-life, feeling she "never was good enough" and did begin the use of alcohol and marijuana at age 12. However, post assault, her combination drug and alcohol use significantly increased as an attempt to "numb myself". This includes taking Lortab (hydrocodone/acetaminophen) and Xanax (alprazolam) at age 18 for a period of time. She had multiple medication trials including Lexapro (escitalopram) and Celexa (citalopram), however she experienced a "noticeable difference" when begun on Prozac (fluoxetine). She had been to two prior rehab programs and although learned some coping skills, she felt that processing the underlying assault had never been done. She and her therapist decided that this was necessary to help achieve future abstinence. The psychotherapy is described as follows: Therapy focused on supporting Patient $\mathrm{Z}$ as she processed her trauma. Relevant topics included patterns of victimization, repetition compulsion as a defense mechanism, appreciation of her anxious attachment style, and her need to disengage from her emotions through self-medication. Her beginning affect was very restricted and her level of participation was minimal; but as rapport developed, she was able to process her trauma more fluidly. The post session anxiety was very strong.

Medication wise, Neurontin (gabapentin), initially at $300 \mathrm{mg}$ three times a day and then up to $1200 \mathrm{mg}$ three times a day was added to try to quell the generalized anxiety. With a multimodal approach including coping skills, exercise, relaxation and visualization exercises, she experienced less anxiety overall. However, the breakthrough anxiety post therapy or when thinking about the assault or current relationship stressors as connected to the assault led to increased anxiety to the point where she was thinking of leaving Against Medical Advice (AMA).

She had tried Vistaril (hydroxyzine) without positive effects, and felt that even low dose Seroquel (quetiapine; 25 to $50 \mathrm{mg}$ range) led to sedation. Therefore, the option of the compounded PanX $\mathrm{X}^{\infty}$ drug combination of atenolol $25 \mathrm{mg}$ and scopolamine $\mathrm{HBr} 0.2 \mathrm{mg}$ in an orally-disintegrating tablet form (compounded by Pine Pharmacy, NY) was given for breakthrough anxiety. The patient provided written informed consent to participate in a physician-sponsored study to try the commercially-available compounded medication. She took the medication on three different occasions and experienced beneficial effects. Prior to taking, she was experiencing anxiety on a 9-10/10 scale. She endorsed anxiety, agitation, panic, increased heart rate, shaking, shortness of breath, and fears of losing control or going crazy. She would endorse these symptoms lasting 45 minutes in a severe state with ongoing anxiety negatively affecting the rest of the day. She took the PanX combo drug by oral mucosal delivery. It took about 2 minutes to fully dissolve and she noted a significant calming effect after about 25 to 30 minutes. The major symptoms which resolved include her elevated heart rate, anxiety (anxiousness), panic, agitation, and trembling. She described the anxiety dropping to a level of 3 out of 10 . She stated this was a similar response with prior use of Klonopin (clonazepam) (3/10), and close to that of a Xanax (alprazolam) (1/10). She denied any sedation, dizziness, fatigue, blurred vision, or confusion. Her only side effect was dry mouth.

Regarding third-party objective assessments, upon discussion with staff who were monitoring her, they did not notice any enlarged pupils, and believed she was calm and interacting well with other patients. Table 1 is a reflection of her blood pressure and heart rate monitored prior to the drug combo, and about 1 and 4 hours post-drug treatment on three different occasions of acute anxiety. Suppression of heart rate by $16-28$ percent at one hour post-drug administration was apparent on all three occasions. Aside from the first trial, her blood pressure remained essentially unchanged.

Overall, Patient $\mathrm{Z}$ did feel much better and felt she was able to continue to process her trauma. She felt she had confidence that she could now better manage her anxiety. The patient perceived a beneficial effect of the drug combo that persisted for three to four hours. The PanX $\mathrm{X}^{\circ}$ combo allowed her to know that she would be able to safely reduce the anxiety after a psychotherapy session, which allowed for the ability to go deeper in sessions. This resulted in improved symptoms as well as better connection and trust with staff and other patients. She endorsed less guilt and shame, and had improved self-esteem.

\section{Discussion/Conclusion}

Dealing with anxiety that is trauma-related or post-therapy is very challenging. If the symptoms are not managed, often patients will stop therapy or there will be a delay in processing the trauma. In Patient Z's case, two other rehab treatments decided to forego processing the trauma. Therapeutic interventions included cognitive behavioral, problem or solution focused, and supportive therapies. Although these are all considered valid approaches, in Patient Z's case it did not result in abstinence from polysubstance use as a coping and numbing tool.

The PanX $\mathrm{X}^{\circ}$ combination drugs were developed as alternatives to prn benzodiazepines, without the use of any addictive active ingredients or Controlled Substances [3]. This case report provides 
clinical evidence of rapid and sustained anxiolytic effect for hours, and on multiple occasions of acute anxiety. This article describes a physician-sponsored study with a novel beta blocker plus scopolamine combination, which was beneficial in a situation that most likely a prn benzodiazepine would have been utilized. Atenolol was selected for several reasons. It has been shown to be beneficial in performance anxiety (social anxiety disorder), alcohol withdrawal, and flight phobia [4-8]. It is beta- 1 selective and is thus preferred (over a non-selective beta blocker) for patients with asthma or COPD. Scopolamine is a potent muscarinic receptor antagonist that was known a century ago to exhibit a calming effect [9].

This is the first case report documenting a trial of any beta blocker - antimuscarinic combination product regarding anxiolytic efficacy and timing, cardiovascular effects, and side effects specifically focused on the anxiety that can accompany the psychotherapy process, and in this case a patient with PTSD. Of note, the patient did experience some dry mouth, which is expected with a sublingual product containing scopolamine that is known to inhibit salivation. Also, her elevated heart rated decreased on all three occasions of drug administration, suggestive of an effect of the beta blocker, and once this included a period of bradycardia, which from her subjective perspective was not apparent. This does reflect the importance of recognizing as with any beta blocker, it is recommended that heart rate and blood pressure be occasionally monitored especially in patients who have lower heart rates or blood pressures at baseline.

There are three FDA-approved approaches for short-term treatment of acute anxiety, most notably benzodiazepines, but also buspirone and hydroxyzine. However, the effectiveness of the latter two agents for acute anxiety states is debatable, with limited published evidence and a general perception by many healthcare providers and patients that buspirone and hydroxyzine aren't effective. Buspirone is thought to be more effective for prevention of anxiety states, similar to the prophylactic Serotonin Reuptake Inhibitors (SSRIs). Therefore, the patented PanX $\mathrm{X}^{\circ}$ drug combinations are a promising new class of anxiolytics, deserving of additional case reports and clinical research. This is the only new class of prn fast-acting anxiolytic treatments (as opposed to slow-acting prophylactics) to be developed and enter human use in many decades. Plus, these drugs have the benefit of not including any addictive active ingredients.

Further studies could address whether the atenolol - scopolamine combo or other beta blocker - antimuscarinic combos are effective for breakthrough anxiety, other acute anxiety episodes, and/or as a regularly dosed oral medication for anxiety disorders. Further research could focus on treatment effectiveness in situational versus state anxiety, social anxiety disorder (e.g., performance anxiety), generalized anxiety disorder, PTSD, panic attacks, specific phobias (e.g., agoraphobia, travel phobia, medical procedures), anxiety associated with alcohol or drug addiction and withdrawal, and perhaps OCD. There are ample medical needs for new effective, nonaddicting, prn anxiolytics, especially for acute anxiety episodes and instead of addictive benzodiazepines.
In view of this PTSD patient's beneficial anxiolytic results following psychotherapy, it would be of interest to determine if the drug combination could be effective during versus after psychotherapy or cognitive behavioral therapy. For instance, the beta blocker propranolol has been used with success in conjunction with CBT for specific phobias [10]. In view of the risks of long-term benzodiazepine use, other novel anxiolytics, such as the beta blockerantimuscarinic combinations, are needed and welcome in this field.

\section{Acknowledgements \& Declarations}

The authors are grateful for the assistance of Robert Waldman, MD, Internal Medicine/Addiction Specialist. Dr. Benjamin is a consultant to TPD LLC. Ms. Mollner has no declarations. Dr. Dooley is an employee and shareholder in Trends in Pharma Development LLC (TPD LLC; www.PanX.us). PanX combination drugs are exclusive to TPD LLC and are protected by US patents (US 9,446,030; US9,517,231;US 9,616,052),international patent applications, and a US Registered Trademark. Compounded drugs are not US FDA approved, but are subject to Section 503A (or 503B) of the FD\&C Act of 2013 (DQSA).This research did not receive any specific grant. No financial support was provided to either the patient or the clinic for participating in the physician-sponsored study.

\section{References}

1. Rozental A, Kottorp A, Boettcher J, Andersson G, Carlbring P. Negative Effects of Psychological Treatments: An Exploratory Factor Analysis of the Negative Effects Questionnaire for Monitoring and Reporting Adverse and Unwanted Events. PLoS One. 2016; 11: e0157503.

2. Davis MA, Lin LA, Liu H, Sites BD. Prescription Opioid Use among Adults with Mental Health Disorders in the United States. J Am Board Fam Med. 2017; 30: 407-417.

3. Dooley TP. Treating anxiety with either beta blockers or antiemetic antimuscarinic drugs: A review. Mental Health in Family Medicine. 2015; 11: 89-99.

4. Neftel KA, Adler RH, Kappeli L, Rossi M, Dolder M, Kaser HE, et al. Stage fright in musicians: a model illustrating the effect of beta blockers. Psychosom Med. 1982; 44: 461-469.

5. Kraus ML, Gottlieb LD, Horwitz RI, Anscher M. Randomized clinical trial of atenolol in patients with alcohol withdrawal. N Engl J Med. 1985; 313: 905909.

6. Horwitz RI, Gottlieb LD, Kraus ML. The efficacy of atenolol in the outpatient management of the alcohol withdrawal syndrome. Results of a randomized clinical trial. Arch Intern Med. 1989; 149: 1089-1093.

7. Gottlieb LD, Horwitz RI, Kraus ML, Segal SR, Viscoli CM. Randomized controlled trial in alcohol relapse prevention: role of atenolol, alcohol craving, and treatment adherence. J Subst Abuse Treat. 1994; 11: 253-258.

8. Ekeberg O, Kjeldsen SE, Greenwood DT, Enger E. Effects of selective beta-adrenoceptor blockade on anxiety associated with flight phobia. J Psychopharmacol. 1990; 4: 35-41.

9. Houde A. Scopolamine: A Physiological and Clinical Study. The American Journal of Clinical Medicine. 1906; 13: 365-367.

10. Soeter M, Kindt M. An Abrupt Transformation of Phobic Behavior after a PostRetrieval Amnesic Agent. Biol Psychiatry. 2015; 78: 880-886.
Ann Depress Anxiety - Volume 4 Issue 2 - 2017

ISSN : 2381-8883 | www.austinpublishing group.com

Benjamin et al. ( ) All rights are reserved
Citation: Benjamin A, Mollner A and Dooley TP. Atenolol - Scopolamine Combination Drug Decreases Acute Anxiety Post Trauma Therapy Session. Ann Depress Anxiety. 2017; 4(2): 1086. 